\title{
Numerical Simulations of Complex Multiphase Flows: Opportunities and Challenges
}

\author{
Gretar Tryggvason \\ Charles A. Miller Jr. Distinguished Professor \\ Head, Department of Mechanical Engineering \\ 223 Latrobe Hall, 3400 N. Charles Street \\ Johns Hopkins University
}

\begin{abstract}
Humans live at the interface between water and air and it is therefore not surprising that multiphase flows are important to many natural and industrial processes. Modeling such flows and developing predictive capabilities is therefore of critical importance. Direct numerical simulations (DNS), where the governing continuum equations are solved accurately for systems that are large enough so that meaningful statistical quantities can be computed, are rapidly becoming routine, at least for systems where the physics is simple enough, such as disperse flows of bubbles and drops. This success opens up new challenges and possibilities. Two of those are discussed here. First of all, while DNS yield enormous insight, they are limited to very small systems and for most relevant processes we need coarser or reduced order models, where small scale processes are represented in an average or statistical way. Often, the presence of a sharp interface is a critical aspect of the flow and retaining an interface when the flow is coarsened it likely to yield improved predictions. We will discuss recent efforts to do so. Secondly, success in modeling relatively simple flows, such as those with bubbles and drops is opening up the possibility of studying much more complex flows, where many competing physical processes take place simultaneously on vastly different time and length scales. We will discuss recent work on modeling three phase flows, as found in a variety of flotation processes used to sort material in the mineral industry, recycling and water remediation.
\end{abstract}

